\title{
Influence des conditions de culture sur l'excrétion d'une mycotoxine par quelques souches de Penicillium roqueforti
}

\author{
par \\ Marie-Thérèse PIVA, J. GUIRAUD, J. CROUZET \\ et P. GALZY \\ Laboratoire de Biochimie Appliquée et Laboratoire de Microbiologie, \\ Sciences et Technologies des Industries Alimentaires, \\ Université des Sciences et Techniques du Languedoc \\ 34060 Montpellier cedex (France)
}

La présence d'une mycotoxine excrétée par Penicillium roqueforti a été mise en évidence en 1973 par Ru-dong Wei et al. [1]. La substance incriminée s'est révélée être un dérivé époxydique d'une $\Delta$ 1-9 optalone 2 de formule [2] :

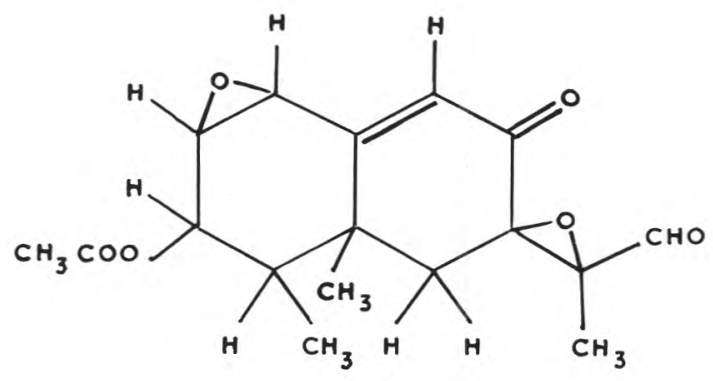

Elle a été désignée par Ru-dong Wei sous le nom de P.R. toxine.

Les doses toxiques pour le rat sont de $11 \mathrm{mg} / \mathrm{kg}$ par voie intrapéritonéale et de $115 \mathrm{mg} / \mathrm{kg}$ par voie orale [1]. Bien que cette toxicité soit faible, nous avons entrepris l'étude systématique des conditions de production de cette toxine. 


\section{MATERIEL ET METHODES}

\section{Matériel biologique}

Trois souches ont été utilisées :

- CBS 221-30 qui est la souche NRRL 849 utilisée par Ru-dong Wei.

- A 1 et A 6 qui sont des souches industrielles utilisées en fromagerie.

\section{Conditions de culture}

Les cultures ont été conduites soit en erlenmeyers remplis au $1 / 6$ de leur volume et agités ( 66 oscillations $/ \mathrm{mn}$; amplitude $7 \mathrm{~cm}$ ), soit en boîtes de Roux contenant $100 \mathrm{ml}$ de milieu. Dans le premier cas, la culture est immergée et sera désignée par la lettre I. Dans le deuxième cas, le mycélium est aérien car il pousse en surface ; la culture sera désignée par la lettre A.

Le milieu de base est de l'extrait de levure Difco 2 p. 100, il est additionné de substrat carboné (glucose, saccharose ou acide lactique) à des concentrations variables. Le pH est ajusté à 4,5 par de l'acide chlorhydrique. Ces milieux sont désignés dans le tableau 1 par l'initiale du substrat carboné.

Dans quelques cas, le milieu a reçu un tampon (milieu $\mathrm{T}$ ) : solution $\mathrm{M} / 5$ de phosphate monopotassique $(\mathrm{pH} 4,5)$. Nous avons également étudié l'effet de l'addition de sel $\mathrm{NaCl} 10$ p. 100).

Les ensemencements ont été réalisés en apportant $10^{4}$ à $10^{5}$ conidies par ml. La souche est cultivée en boîte de Pétri sur milieu Potato-Dextrose-Agar Difco. Les conidies sont recueillies par lavage à l'eau stérile additionnée de Tween 80 à 0,05 p. 100 .

Les cultures d'un volume global de 21 ont été conduites pendant $15 \mathrm{j}$ à $25^{\circ} \mathrm{C}$.

\section{Extraction de la toxine}

Le milieu de culture est filtré plusieurs fois sur verre fritté $n^{\circ} 1$ puis $\mathrm{n}^{\circ} 4$ de façon à éliminer le mycélium et les conidies. Le filtrat recueilli d'un volume de 1,750 l, est extrait cinq fois par 1,2 l de chloroforme Merck. L'extrait chloroformique est évaporé jusqu'à obtention d'un résidu huileux coloré en brun qui va subir des purifications successives par chromatographie sur colonne.

Le résidu dissous dans le minimum de chloroforme est déposé sur une colonne de $3 \mathrm{~cm}$ de diamètre et $35 \mathrm{~cm}$ de longueur contenant 
$20 \mathrm{~g}$ de silice Kieselgel 60 (70 à 230 mesh) Merck et élué par du chloroforme. Une fraction unique de $400 \mathrm{ml}$ est recueillie et évaporée à sec.

Le résidu de cette fraction est purifié à travers une $2^{\mathrm{e}}$ colonne de $3,5 \mathrm{~cm}$ de diamètre et $100 \mathrm{~cm}$ de longueur contenant $240 \mathrm{~g}$ de silice du même type que précédemment et élué par du chloroforme/ méthanol 96/4. Des fractions de $10 \mathrm{ml}$ sont recueillies avec un débit de $0,5 \mathrm{ml}$ par minute. Les fractions 40 et 76 sont recueillies et évaporées à sec.

Une $3^{e}$ purification est réalisée à l'aide d'une colonne de $3,2 \mathrm{~cm}$ de diamètre et $100 \mathrm{~cm}$ de longueur contenant $80 \mathrm{~g}$ de Sephadex LH 20 (Pharmacia, France). Le résidu précédent est dissous dans $2 \mathrm{ml}$ de chloroforme/éther de pétrole $60 / 40$ et élué avec le même solvant avec un débit de $0,25 \mathrm{ml}$ par minute. Les fractions de $5 \mathrm{ml} \mathrm{n}^{\circ} 25$ à 35 sont recueillies et le solvant éliminé.

Le résidu obtenu contient de la P.R. toxine.

\section{Identification de la toxine}

\section{- Chromatographie en couche mince}

La détection de la toxine au cours de la purification se fait par chromatographie en couche mince sur des plaques fabriquées par nous-mêmes avec de la silice Kieselgel 60 pour CCM Merck. La technique utilisée est celle de Ru-dong Wei [1], la migration est réalisée à l'aide de chloroforme/méthanol 96/4.

En lumière U.V., la P.R. toxine apparaît sous forme d'une tache fluorescente bleue qui vire en quelques minutes au gris-vert. Lorsque les plaques sont soumises à une pulvérisation d'acide sulfurique à 50 p. 100, la P.R. toxine vire au jaune et devient visible à la lumière du jour.

\section{- Spectrographie de résonance magnétique nucléaire}

L'identification de la toxine a été réalisée par spectrographie RMN des protons à l'aide d'un appareil Varian A 60. Pour effectuer la mesure, 30 à $50 \mathrm{mg}$ du dernier résidu sont mis en suspension dans $0,5 \mathrm{ml}$ de chloroforme deutéré $\left(\mathrm{CDCl}_{3}\right)$. La résonance des protons se manifeste par l'apparition de signaux dont on repère le déplacement chimique par rapport à un signal témoin obtenu à l'aide de TMS (tétraméthylsilane), standard, arbitrairement choisi, et qui est utilisé ici en référence interne. La fréquence de résonance des différents protons dépend de la nature du proton et de la proximité des liaisons chimiques environnantes. De plus, par suite de phénomènes de couplage spin-spin des protons, un groupe de protons équivalents donne un signal qui est dissocié en $n+1$ multiplets sous l'action 

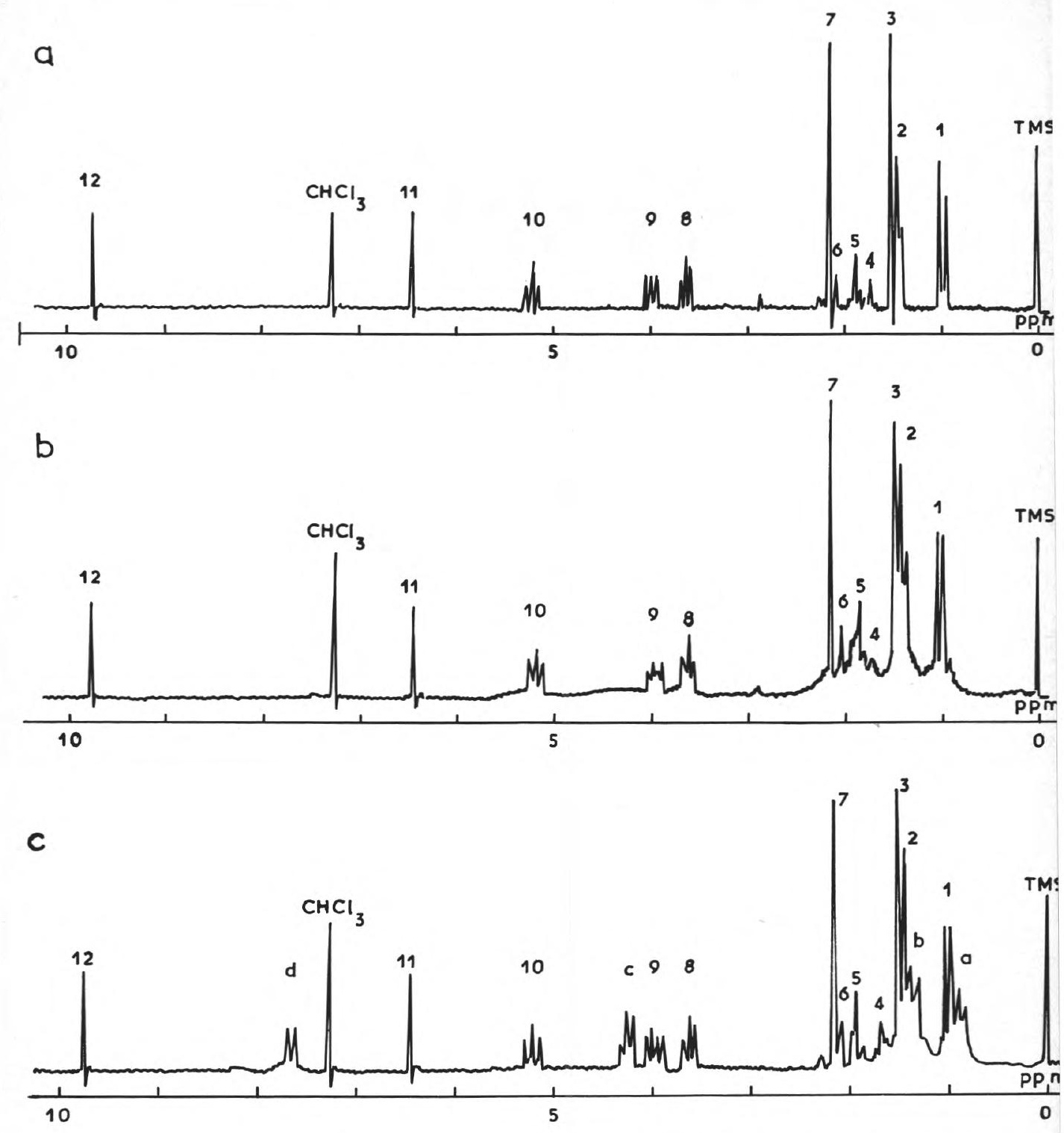

d

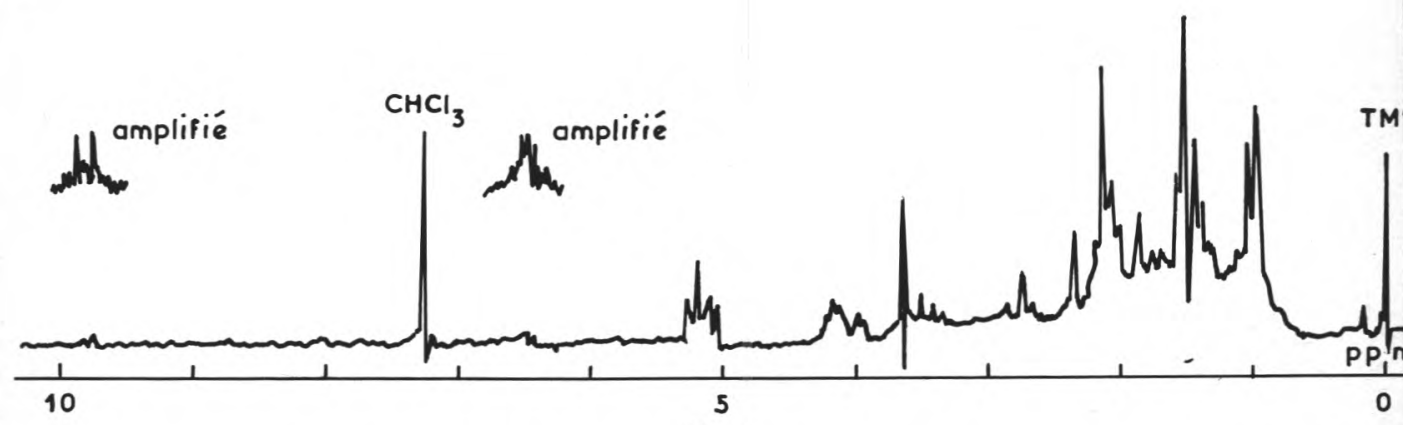

fig. 1

Spectre RMN des résidus recueillis à partir de diverses souches

a) Spectre obtenu par Ru-dong Wei

c) Spectre du résidu : souche A 1

b) Spectre du résidu : souche CBS 221-30 milieu A/S 15 milieu A/S 15

d) Spectre du résidu : souche $\mathrm{A} 6$ milieu A/S 15/T 
de $\mathrm{n}$ protons équivalents voisins. Il est donc possible, dans ces conditions, d'obtenir des renseignements sur la structure de la molécule. La surface des pics obtenus est proportionnelle à la concentration du produit étudié.

La figure 1 a représente le spectre obtenu par Ru-dong Wei pour la P.R. toxine pure. Nous avons numéroté les signaux ou groupes de signaux (doublets, triplets, etc.) de 1 à 12 afin de faciliter la compréhension des spectres.

Les doublets 1 et 2 ainsi que le pic 3 correspondent aux protons de groupements méthyl. Le pic 7 correspond aux protons d'un acétoxyméthyl, le pic 11 à un proton éthylénique, le 12 à un proton aldéhydique. Les pics ou groupes de pics $4,5,6,8,9,10$, correspondent aux protons restants. Outre ces 12 signaux, il apparaît sur les spectres celui correspondant au témoin TMS et celui correspondant aux traces ce chloroforme $\left(\mathrm{CHCl}_{3}\right)$ présents dans le chloroforme deutéré $\left(\mathrm{CDCl}_{3}\right)$.

Les pics 11 et 12 ont retenu notre attention compte tenu du fait qu'ils se situent dans des zones relativement dépourvues d'autres pics, ce qui facilite l'identification de la toxine.

La détermination quantitative a été faite par pesée de la toxine obtenue à l'état pur. Lorsque le spectre RMN a révélé la présence d'impuretés, la quantité de toxine a été calculée en mesurant la surface du pic 11 sur le spectre du résidu impur et en se référant à celle du pic correspondant sur le spectre de la toxine pure.

\section{RESULTATS EXPERIMENTAUX}

\section{Influence des conditions de milieu sur la croissance}

Le tableau 1 indique les résultats relatifs à la croissance des trois souches ; pH final et récolte de mycélium exprimée en grammes de matière sèche par litre. Dans des conditions de culture identiques, les trois souches donnent des résultats comparables : la souche A 1 pousse légèrement mieux que les deux autres.

La croissance sur acide lactique reste toujours limitée quelle que soit la concentration en substrat utilisée. L'utilisation de l'acide lactique provoque une rapide augmentation de $\mathrm{pH}$ au-delà de 8 et le $\mathrm{pH}$ obtenu bloque la croissance. Les tampons utilisés ne nous ont pas permis d'éviter cette augmentation du $\mathrm{pH}$.

En présence de saccharose, la croissance est toujours plus forte qu'en présence d'acide lactique. Le $\mathrm{pH}$ s'élève aux environs de 8 sans jamais atteindre cette valeur lorsque la concentration en glucide est faible (1,5 p.100). L'activité protéolytique du mycélium 
$T A B L E A U$

Etude de la croissance des trois souches de Penicillium roqueforti dans diverses conditions de culture

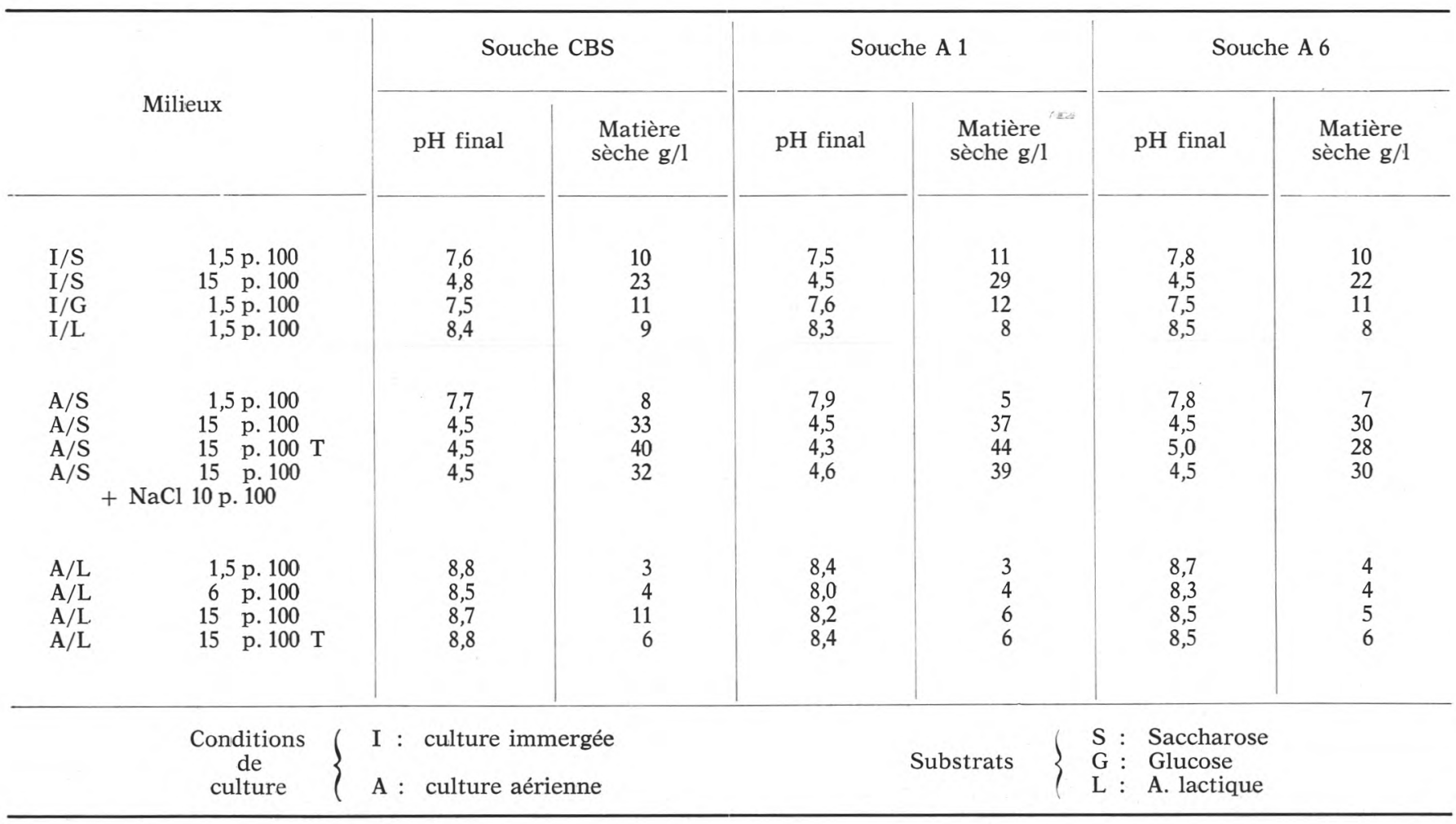


libère de l'ammoniac après épuisement du glucide. Le $\mathrm{pH}$ final est au contraire bas ( 4,3 à 5$)$ lorsque la concentration en glucide est élevée (15 p. 100), et la croissance abondante. Dans ce cas, le pH se maintient à ces valeurs pendant les 15 jours de culture.

Nous avons observé que le mycélium reste d'un vert franc ou légèrement bleuté selon la souche lorsque le $\mathrm{pH}$ est inférieur à 6 . Au-dessus de cette valeur, le mycélium devient grisâtre puis au-delà de $\mathrm{pH} \mathrm{7,} \mathrm{brunâtre.}$

En culture immergée, la croissance sur saccharose 15 p. 100 est un peu moins bonne qu'en culture en surface. Il est possible que l'aération des cultures immergées soit insuffisante pour assurer la croissance maximale.

\section{Influence des conditions de milieu sur la production de mycotoxine}

La mycotoxine n'a jamais été mise en évidence dans les extraits obtenus à partir des cultures immergées. Pourtant sur le milieu à base de saccharose 15 p. 100, la production de mycélium était importante. Selon la nature du substrat, nous avons obtenu des $\mathrm{pH}$ finaux élevés (\# 8) ou faibles $(4,5)$. Il est possible que l'aération soit insuffisante pour la production de mycotoxines dans ces cultures.

La mycotoxine n'a jamais été mise en évidence dans les cultures sur acide lactique comme source de carbone. L'absence d'excrétion de la mycotoxine peut s'expliquer, soit par la faiblesse de la croissance, soit par l'élévation du $\mathrm{pH}$ qui dépasse rapidement la valeur de 8 , soit par la nature même du substrat.

La mycotoxine apparaît dans certains cas lorsque la culture est réalisée sur milieu saccharose. Il faut discuter tout d'abord le cas des souches CBS 221-30 et A 1. En présence de saccharose 1,5 p. 100, la toxine n'apparaît pas, soit parce que la croissance est faible, soit parce que le $\mathrm{pH}$ en fin de culture est trop élevé. La mycotoxine ne se forme que dans les conditions déjà décrites par Ru-dong Wei [1], en présence de saccharose $15 \mathrm{p} .100$. La production est de $2 \mathrm{mg}$ de toxine par gramme de mycélium formé pour la souche CBS et de $6 \mathrm{mg}$ pour la souche A 1. La formation de toxine n'a pas été mise en évidence après addition dans le milieu de culture de tampon phosphate ou de $\mathrm{NaCl} 10$ p. 100.

Les figures $1 \mathrm{~b}$ et $1 \mathrm{c}$ représentent les spectres RMN correspondant aux extraits obtenus à partir des souches CBS et A 1 après culture aérienne sur saccharose 15 p. 100.

Le spectre $1 \mathrm{~b}$ correspondant à la souche CBS est identique à celui obtenu par Ru-dong Wei (1a) : la toxine obtenue est pure. 
Le spectre $1 \mathrm{c}$ correspondant à la souche A 1 présente quelques différences : il faut noter, en effet, la présence de pics assez importants a, b, c, d, qui viennent s'ajouter à ceux caractéristiques de la toxine. Ces pics proviennent d'impuretés qui colorent d'ailleurs le résidu en brun. Nous avons vérifié que les fractions colorées précédant la P.R. toxine lors de l'élution présentent un spectre où n'apparaissent que les pics $a, b, c, d$. Une partie de cette substance est vraisemblablement en mélange avec la P.R. toxine sans cependant géner son identification.

Quelques conclusions se dégagent de ces résultats. La mycotoxine ne se forme pas en culture immergée : il est probable que sa formation exige un métabolisme fortement aérobie. En effet, dans des conditions de culture parfaitement identiques en dehors de l'aération, on n'observe en culture agitée (I/S 15), aucune formation de toxine alors que dans le cas de la culture en surface (A/S 15), on note la formation de toxine. Les récoltes de mycélium obtenues en culture immergées sont dans ce cas importantes et auraient dû permettre de déceler la toxine.

La mycotoxine n'a jamais été mise en évidence dans les cultures où le $\mathrm{pH}$ final est élevé. Dans ce cas, les populations finales ne sont pas généralement très abondantes mais la méthode analytique utilisée aurait dû permettre de mettre la toxine en évidence si elle avait été formée.

L'apparition de toxine ne peut être décelée que dans les cultures en surface présentant une croissance abondante et un $\mathrm{pH}$ final bas $(4,5)$, proche du $\mathrm{pH}$ initial. Il n'est pas possible d'affirmer que la présence d'un glucide est indispensable, mais on sait que certaines mycotoxines (aflatoxines) se forment préférentiellement lorsque la quantité de glucide est importante dans le milieu.

L'absence de toxine en culture sur lactate peut être liée à la nature du substrat ou à l'augmentation du $\mathrm{pH}$ liée à son métabolisme. De toute façon, que la cause soit directe ou indirecte, il ne se forme pas de toxine sur acide lactique.

Il est plus difficile d'expliquer pourquoi la toxine ne se forme pas sur le milieu à 15 p. 100 de saccharose chaque fois qu'une addition (phosphate ou $\mathrm{NaCl}$ ) est réalisée. Peut-être la force ionique du milieu intervient-elle ? Pourtant sur ces milieux, la croissance est bonne et le $\mathrm{pH}$ final reste bas.

La souche A 6 a un comportement particulier. Aucune trace de toxine n'a été mise en évidence sur le milieu Ru-dong Wei ni dans la plupart des conditions d'expériences. Une exception toutefois, un extrait a été obtenu sur milieu saccharose 15 p. 100 additionné de phosphate monopotassique $\mathrm{M} / 5$. La figure $1 \mathrm{~d}$ représente le spectre RMN de cet extrait. A la sensibilité normale de l'appareil, les pics 11 
et 12 caractéristiques du produit n'apparaissent pas. Si l'on augmente l'amplitude de l'enregistrement, il apparaît dans le bruit de fond des signaux qui pourraient correspondre aux pics 11 et 12 et laisser supposer la présence de P.R. toxine. Il n'est pas cependant possible de caractériser cette toxine de façon absolue. Il est permis de penser que si la P.R. toxine est présente, ce n'est qu'à l'état de traces. Cependant, l'allure générale du spectre, en dehors de l'absence de ces deux pics, laisse supposer l'existence d'un composé ayant un squelette voisin de celui de la toxine : analogue structural, précurseur ou produit de dégradation.

A ce stade de travail, on ne peut affirmer ou infirmer catégoriquement la présence de la P.R. toxine dans les cultures de la souche A 6.

\section{CONCLUSION}

La production de la P.R. toxine décrite par Ru-dong Wei est très étroitement dépendante des conditions de culture. Il est difficile d'expliquer au niveau métabolique les raisons de l'action des divers facteurs étudiés. Cependant, il est intéressant de noter que les facteurs susceptibles d'empêcher la formation de la P.R. toxine sont : l'insuffisance d'aération, la présence de chlorure de sodium ou d'un facteur augmentant la force ionique, l'augmentation du $\mathrm{pH}$ à des valeurs voisines de la neutralité. De plus, la mycotoxine ne semble se former que si le milieu est riche en sucre. Il faut rappeler que les conditions défavorables à la production de la P.R. mycotoxine sont, pour la plupart, réunies dans les fromages du type bleu : aération faible, absence de glucide, présence de $\mathrm{NaCl}$, et même tendance à l'augmentation du $\mathrm{pH}$.

S'il était permis de s'inquiéter de l'excrétion d'une mycotoxine chez une souche de Penicillium roqueforti dans des conditions de culture in vitro bien particulières, nos résultats montrent qu'il est hautement improbable qu'une souche de Penicillium puisse secréter cette substance dans les conditions de l'affinage des fromages.

De plus, l'une des souches étudiées, la souche A 6, semble incapable de former la P.R. toxine. Il est donc possible de sélectionner des souches ne présentant aucun risque d'excrétion de P.R. toxine, mème dans des conditions très favorables à sa production.

\section{Rés u m é}

La production de P.R. toxine par certaines souches de Penicillium roqueforti n'intervient que dans des conditions de culture très parti- 
culières (milieu saccharosé à 15 p. 100) qui sont éloignées de celles rencontrées dans les fromages. Toutes les souches ne produisent pas la P.R. toxine.

\section{S u $\mathrm{m} \mathbf{m}$ a r y}

Production of P.R. toxin by some strains of Penicillium roqueforti occurs only in very specific conditions of culture (concentration of sucrose in the medium: 15 p. 100). This conditions do not occur in the cheese. All the strains do not produce P.R. toxin.

\section{Remerciements}

Nous remercions la Confédération Générale de Roquefort de nous avoir confié ce travail et fourni les moyens de le mener à son terme.

Nous remercions également $M$. Solanet, Directeur de la Société des Caves, ainsi que Mlle Rivemale et M. Assenat du Laboratoire de la Société des Caves, pour la fourniture des souches de Penicillium, pour leurs informations et conseils.

Reçu pour publication en janvier 1976.

\section{Bibliographie}

[1] Wei (Ru-dong), Still (P. E.), Smalley (E. B.), Schnoes, (H. K.), Strong (E. M.). (1973). - Isolation and rartial characterization of a mycotoxin from Penicillium roqueforti, Applied Microbiology, 25, 111-114.

[2] Wei (Ru-dong), Schnoes (H. K.), Hart (P. A.), Strong (F. M.) (1975). - The structure of P.R. toxin, a mycotoxin from Penicillium roqueforti. Tetrahedron, 31, 109-114. 